\title{
SANTÉ PUBLIQUE ET PARLEMENTS AU XVIIIe SIÈCLE; LA LIMITE DU CHAMP D'ACTION DE LA POLICE SANITAIRE. ANALYSE DE L'ARRÊT DU CONSEIL D'ÉTAT DU ROI DU 14 SEPTEMBRE 1720 AU SUJET DE LA MALADIE CONTAGIEUSE DE LA VILLE DE MARSEILLE
}

\author{
SALUD PÚBLICA Y PARLAMENTOS EN EL SIGLO \\ XVIII; LA LIMITACIÓN DEL CAMPO DE ACCIÓN DE LA \\ POLICÍA SANITARIA. ANÁLISIS DE LA RESOLUCIÓN \\ DEL CONSEJO DE ESTADO DEL REY DE 14 DE \\ SEPTIEMBRE DE 1720 EN RELACIÓN A LA EPIDEMIA \\ CONTAGIOSA DE LA VILLA DE MARSELLA
}

\author{
JULIE CADDEO 1
}

Recibido: 10/12/2019

Aceptado: 28/01/2020

SOMMAIRE: I.- Une décision unilatérale opérant un contrôle de proportionalité. II.- Une mesure portant la distinction entre prevention et lutte contre la maladie contagieuse. III.- Une mesure ne remettant pas en cause la compétence sanitaire des parlements de province.

SUMARIO: I.- Una decisión unilateral operando un control de proporcionalidad. II.- Medida que conlleva la distinción entre prevención y lucha contra la enfermedad contagiosa. III.- Medida que no cuestiona la competencia sanitaria de los parlamentos de provincia.

SUMMARY: I.- A unilateral decision operating a proportionality control. II.- A measure bringing the distinction between prevention and control against contagious diseases. III.- A measure that does not challenge the health competence of the parliaments of province.

Résumé: Lorsque l'on s'attarde sur l'arrêt du 14 septembre 1720 promulgué par Louis XIV concernant la peste qui sévit à Marseille, on trouve de nombreux renseignements en ce qui concerne les attributions des parlements de provinces en matière de santé publique. L'étude de cette mesure nous permet donc de porter un nouveau regard sur le rôle des institutions locales au dix-huitième siècle.

Mots-clés: Ancien Régime - Santé public - Réglementation applicable - Normes médicales et de police - tribunal local

Resumen: Cuando nos detenemos en el Decreto de 14 de septiembre de 1720, promulgado por Luis XIV, en relación a la peste que asolaba Marsella, encontramos numerosas enseñanzas en relación a las atribuciones de los Parlamentos de provincia en

1. Doctorante en histoire du droit à l'Université de Nantes, UMR-CNRS 6797. E-mail: julie.caddeo@outlook.fr 
materia de salud pública. El estudio de esta medida nos permite otear un nuevo enfoque sobre el rol de las instituciones locales en el siglo dieciocho.

Palabras clave: Antiguo Régimen - salud pública - regulación aplicable - normativa médica y de policía - tribunales de primera instancia

Abstract: When we take a look at the Louis XIV's arrest of $14^{\text {th }}$ septembre 1720 about the contagious disease in the city of Marseille, beyond this measure, it is quite interesting to see how many informations we can find in matters of local Courts's attributions in France about health practices. In this aspect, the study of this arrest allow us to focus on the local intitutions role in the $18^{\text {th }}$ century in France of Ancien Régime.

Key words: Ancien Régime - public health -applicable regulation - medical norm and police - locals Court

La prise de conscience, durant la seconde moitié du XVIII ${ }^{\mathrm{e}}$ siècle $^{2}$, amenant l'État à prendre en charge la santé publique ne résulte pas d'une simple magnanimité mais d'une juste nécessité. Pour cela, il nous faut revenir sur les fléaux qui ont marqué le $\mathrm{XVII}^{\mathrm{e}}$ siècle. La principale cause de mortalité est bien connue: la peste. Cette épidémie touche alors une grande partie du royaume de France ${ }^{3}$. Sa diffusion en Europe occidentale est facilitée par le paupérisme des civilisations encore marquées et affaiblies par les famines récurrentes. D'autre part, les acteurs publics n'en sont pas encore au stade d'une prise de conscience totale de la dimension médicale et hygiénique de la calamité. De ce fait, on la considère encore comme une malédiction, une fatalité s'abattant aléatoirement sur les peuples ou s'associant à un châtiment divin. Pourtant, au fur et à mesure des années, les sociétés ne font plus l'expérience individuelle de la maladie et prennent conscience de la dimension collective, et par conséquent, de la nécessité d'une prise en charge à ce même niveau ${ }^{4}$. Ainsi, contre ce fléau, les premières mesures d'une gestion prophylactique de l'épidémie sont mises en place ${ }^{5}$.

2. «En France, dans la seconde moitié du XVIII e siècle, l’État prend définitivement conscience de ses devoirs en matière de santé publique et d'assistance médicale collective», SANCHEZ, S., LECA, A., LUNEL, A. (2014), Histoire du droit de la santé, Les études hospitalières, Collection Intempora, Bordeaux, p. 143.

3 . BARRY, S. (1999), «Bordeaux face à la peste aux XVI et XVII siècles», DELVAILLE, C. (dir.), Comité de lecture du 27 février 1999 de la Société française d'Histoire de la Médecine, p. 305 et CAPLET, E.-A. (1898), La Peste à Lille au XVII siècle, Lille, Le Bigot frères, p. 45. Voir aussi REVEL, J. (1970), «Autour d'une épidémie ancienne : la peste de 1660-1670», Revue d'histoire moderne et contemporaine, tome XVII, n4 4 , octobre-décembre, p. 953.

4 . FAURE, O. (1997), «Les stratégies sanitaires», in GRMEK, M. (dir.), Histoire de la pensée médicale en Occident, Paris, t. II, pp. 279.

5 . Rappelons que par «épidémie», on entend le développement et la propagation rapides d'une maladie contagieuse dans une région où elle sévissait auparavant à l'état endémique, ou au sein d'une population préalablement indemne. Cette définition met en lumière les failles possibles de la prévention : dans le premier cas, cette dernière n'a pas su éviter la diffusion de la maladie qui s'est finalement développée, et dans le second, n’a pas su protéger sa population d'une maladie extérieure. En effet, une épidémie, puisqu'elle perturbe le fonctionnement normal de la société et de l'économie, provoque un désordre ayant 
Ainsi, la santé publique apparaît désormais comme le principal vecteur d'affirmation du pouvoir urbain et marchand, comme elle l'est au siècle suivant pour le pouvoir monarchique. Cette avancée se concrétise au XVIII ${ }^{\mathrm{e}}$ siècle lorsque s'affirme une organisation de la sécurité sanitaire. Pour protéger la santé du peuple, les institutions publiques édictent des normes de santé publique ${ }^{6}$, ces dernières se mettant en place sous différents aspects. L'ambition majeure du roi, représentant du bien commun, est la préservation de la santé. C'est l'objet principal qui apparaît lors de la peste de 1720 et qui se traduit, notamment, par la création des bureaux de santé visibles en Provence ainsi qu'en Bas-Languedoc ${ }^{7}$. Cette intervention va concerner deux domaines d'action principaux: la prévention des maladies et la lutte contre les épidémies, dans lesquels le souverain va tout à la fois diriger les pouvoirs locaux mais aussi leur laisser une marge de manœuvre afin qu'ils exécutent au mieux ces deux missions. Les épidémies déréglant le cours normal de l’activité du royaume, on remarque que cette prise en charge relève aussi d'un souci de maintien de l'ordre.

En effet, en matière sanitaire, l'instrument qui se révèle le plus efficace dans de telles circonstances est la police. Elle se conçoit alors, dans sa dimension normative, comme «le pouvoir ordinaire d'édicter des règles stables pour la conservation et l'avantage du bien public» ${ }^{8}$, c'est un moyen de participation à la gestion du royaume.

des incidences multiples obligeant les autorités à agir. L’épidémie se définit aussi comme une «propagation rapide, dans une région où elle sévissait antérieurement à l'état endémique, au sein d'une population préalablement indemne, d'une maladie. S’oppose à l'endémie définie comme une affection persistante dans une région ou au sein d'une collectivité s'y manifestant de façon constante ou périodique», LECA, A. (2003), «La notion d'épidémie dans l’histoire des idées», Avant-propos au colloque d'Aix-en-Provence des 28-29 novembre 2002, «Le risque épidémique», Actes II Colloque national Droit, Histoire, Médecine et Pharmacie, Collection Droit de la santé, Aix-en-Provence, Presses Universitaires Aix-Marseille, p. 13, v. aussi DELHUMEAU, J. et LEQUIN, Y. (1993), Les malheurs des temps. Histoire des fléaux et des calamités, Paris, Larousse, p. 89 et SOURNIA, J.-C. (1984), Les épidémies dans l'histoire de l'Homme, Paris, Flammarion, p. 46. De plus, «deux ou trois conditions sont nécessaires; il faut un grand nombre d'individus réceptifs, un germe existant et entretenu dans un réservoir, enfin, dans le cas d'une maladie non directement transmissible à l'Homme, un agent de transmission. Sous l'Ancien Régime, où le débat a été longtemps porté sur le caractère contagieux ou non contagieux de la peste, l'épidémie est couramment appelée "contagion"», $\mathrm{V}^{\circ}$ «Épidémie», HIDELSHEIMER, F. (2015), Dictionnaire de l'Ancien Régime, Royaume de France, BELY, L. (dir.), Paris, PUF, p. 494.

6 . Il faut entendre «norme» au sens de norme contraignante émanant des institutions publiques; du latin «norma, æ, f., équerre, règle, loi», GAFFIOT, F. (1934), Dictionnaire latin-français, Paris, Hachette, p. 902. Ainsi «l'existence d'une normativité juridique implique des instituions de création, une finalité à servir et des ressorts», BRUNET F. (2009), «Introduction», ROUSSELET-PIMONT, A., BONIN, P., GARNIER, F. et HUMBERT, M. (dir.), Études d'histoire du droit rassemblées en l'honneur d'Albert Rigaudière, Paris, Economica, p. 16.

7 . Ils sont visibles dès le début du siècle, l'article XI de l'arrêt de 1720 prévoyant que «Lesdits commandants et Intendants, et lesdits officiers municipaux pourront aussi établir des gardes aux portes ou entrées des villes et autres passages qu'ils estimeront convenables, et dans les lieux où ils jugeront que cette précaution pourra être nécessaire». L'article X dispose quant à lui que «Sera établi dans tous les lieux de la Provence, où lesdits commandants et Intendant jugeront à propos de le faire, des Bureaux de santé composés des officiers municipaux et autres principaux habitants qui seront choisis à cet effet; en la forme et manière qui sera prescrite par lesdits commandants et Intendant, pour veiller aux précautions nécessaires pour empêcher la communication du mal contagieux, maintenir la police et la discipline qui seront établies à cet égard, et faire exécuter les ordres qu'ils recevront de leurs supérieurs», Arrêt du Conseil d'État du Roi du 14 septembre 1720 au sujet de la maladie contagieuse de la ville de Marseille, Archives Nationales (A. N.), E 2020, f 155.

8 . La police doit être entendue, au XVIII ${ }^{\mathrm{e}}$ siècle, comme «administration», en ce sens v. MESTRE, J.-L. (1985), Introduction historique au droit administratif, Paris, Presses Universitaires de France, pp. 161- 
Plus qu'une simple modalité d'administration revendiquée par le Parlement et prise en compte par l'intendant ${ }^{9}$, elle est un passage obligé dans la recherche de l'ordre social ${ }^{10}$ et la condition essentielle d'une efficacité de la réglementation à l'échelle locale. Cette police procède de la compétence des parlements ${ }^{11}$, cours provinciales du royaume français titulaires du pouvoir réglementaire ${ }^{12}$.

Malgré le champ d'application très large de la police, le rôle des pouvoirs locaux s’inscrit plutôt dans une optique que l’on qualifie de «préventive» ${ }^{13}$, effectuée en

167 et BOULET-SAUTEL, M. (1980), «Police et administration en France à la fin de l’Ancien Régime. Observations terminologiques : Histoire comparée de l'administration (IVe-XVIII ${ }^{\mathrm{e}}$ siècle)», Actes du XVe colloque historique franco-allemand, Tours 1977, Munich, p. 51. En effet, «police se prend communément pour l'ordre qui s'observe dans une Ville ou dans un lieu pour y maintenir la discipline et empêcher les forfaits et les désordres que les scélérats et les ivrognes y pourraient commettre ou qui pourraient être causés par la débauche ou par le luxe», FERRIERE, C. de (1717), Dictionnaire de droit et de pratique, Paris, p. 325. Avant tout, «il s’agit avec la police, de façonner une société et non plus seulement comme au Moyen Âge, de la conserver : le développement de la police est, d'une certaine manière, la concrétisation du volontarisme qui est véhiculé par l'idée de souveraineté. Son champ d'application est essentiellement urbain : le mot "police" doit être compris dans son sens étymologique évoquant l'ordre dans la cité», SAINT- BONNET, F., SASSIER, Y. (2015), Histoire des institutions avant 1789, Domat Droit Public, 2010, p. 409.

9 . «Dès le XVI ${ }^{\mathrm{e}}$ siècle des conflits s’étaient élevés entre les Parlements et gouverneurs au sujet de la police. Toutefois, il apparut rapidement que ces derniers, malgré le désir qu'ils avaient de préserver leurs privilèges, étaient trop grands seigneurs pour avoir un goût réel pour l'administration. Aussi bien, les Parlements sortirent vainqueurs», PAYEN, P. (1997), Les arrêts de règlement du Parlement de Paris au XVIII ${ }^{e}$ siècle. Dimension et doctrine, Paris, PUF, p. 170. En effet, «n'appartient qu'au Roy, ou à ses Parlements, de faire des règlements qui concernent la police générale et universelle du royaume», DELAMARE, N. (1705), Traité de la police, où l'on trouvera l'histoire de son établissement, les fonctions et les prérogatives de ses magistrats; de toutes les loix et règlements qui la concernent, Paris, J. et P. Cot, t. I, p. 54.

10 .Cette finalité sociale est mise en valeur lorsque le procureur général du roi la définit comme tendant «à la sûreté, à la conservation de la société et des membres qui la composent», Archives départementales d’Ille-et-Vilaine (A.D.I-V.), 1Bf1598, cité par BAREAU, R. (2000), Les arrêts de règlement du Parlement de Bretagne, Thèse, Droit, Rennes I, t. II, p. 371.

11. A propos du détenteur de cette police, P. Payen avertit qu' «il convient de noter que cette police générale n’est pas seulement à un degré au-dessus de celle qui est exercée par les officiers subalternes, elle est d'une nature profondément différente. A l'intérieur du champ géographique infiniment plus limité qui était le leur, ils pouvaient bien être appelés à connaître d'objets identiques à ceux sur lesquels le Parlement étendait sa compétence, ils ne possédaient pas pour autant une portion de la police générale. Cette dernière appartenait au Parlement exclusivement car lui seul possédait, après le roi, la justice au plus haut point dans un territoire fixe», PAYEN, P. (1997), Les arrêts de règlement du Parlement de Paris au XVIII ${ }^{e}$ siècle. Dimension et doctrine, p. 766.

12 . Cette compétence de police des parlements dépend d'une conception ancienne et extensive de la justice. A ce titre, «le pouvoir réglementaire des parlements a deux origines. D’une part, il remonte au Moyen Age où celui qui possède le droit de justice a en même temps celui de "police" [...] D’autre part, le pouvoir réglementaire des parlements repose sur une "délégation des pouvoirs du roi" qui, ne pouvant pas tout connaître, ni pourvoir par des lois à tous les besoins qui se révélaient, avait permis aux cours souveraines d'y suppléer par des règlements obligatoires dans leur ressort», ESMEIN, A. (1912), Cours élémentaire d'histoire du droit français, Paris, L. Larose et L. Tenin, p. 530 cité par BAREAU, R. (2000), p. 10.

13 . Cette spécialisation préventive est mise en valeur à la lumière du rôle de la lieutenance de police. En guise d'exemple de cette spécialisation des pouvoirs locaux en tant qu'autorités publiques chargées de la police préventive on constate, au-delà du cadre breton, que «la Lieutenance générale de police de Paris, au siècle des Lumières, développa une "politique policière de santé" urbaine en direction des habitants. Dépassant la lutte contre les épidémies, elle devint préventive et s’intéressa aux liens entre les êtres humains et leur environnement. Elle mobilisa pour cela des savoirs urbains de pointe, en produisit de 
vue d'une protection sanitaire de la province consistant non en une éradication de la contagion, mais en une réduction de l'exposition des habitants aux dangers que sont les maladies présentes ${ }^{14}$. La prévention sanitaire est alors affaire des pouvoirs locaux, mais, qu'en est-il de la place de ces derniers au sein de la lutte contre les épidémies?

Le Conseil d'État du roi, le 14 septembre 1720, décide alors d'intervenir face à cet excès des parlementaires en dépossédant le Parlement d'Aix, et avec lui l'ensemble des parlements, de leurs attributions dans le domaine sanitaire ${ }^{15}$. Ainsi, il est nécessaire de faire une digression sur le cas aixois où trois principaux points doivent être éclaircis afin de mieux comprendre les raisons pour lesquelles cette mesure atteint les parlements provinciaux et la portée réelle de cette dernière.

D’une part, on admettra que cette décision met en balance deux intérêts (I) tout en opérant une distinction entre les mesures préventives et celles concernant la lutte contre la maladie contagieuse (II). Néanmoins, cette mesure ne remet pas définitivement en cause la compétence des parlements de province dans le champ de l'action sanitaire (III).

\section{UNE DECISION UNILATERALE OPERANT UN CONTROLE DE PROPORTIONALITE}

De ce point de vue, c'est un événement marquant de l'histoire sanitaire française qui éclaircit la compétence ratione materiae restreinte des parlements ${ }^{16}$ : la Peste noire de 1720. Cette dernière éclaire leur éviction précoce dans la lutte contre les épidémies, a fortiori leur rôle essentiellement préventif tout au long du XVIII ${ }^{\mathrm{e}}$ C'est alors plus précisément l'intervention royale concernant le Parlement d'Aix qui impacte le rôle de tous les parlements du royaume. Ainsi, lors de cet épisode de peste débutant en 1720, le dispositif provençal habituel fut mis en place; le Parlement d'Aix ${ }^{17}$

nouveaux, et intégra dans sa sphère la Société Royale de Médecine, qui lui permit d’agir directement sur la matérialité urbaine pour améliorer la santé des Parisiens», VIDONI, N. (2015), "Protéger la santé des Parisiens au XVIII ${ }^{\mathrm{e}}$ siècle: savoirs urbains et action policière», Histoire, médecine et santé, nº6, p. 97.

14 . Cette distinction entre lutte préventive et lutte effective en tant qu'éradication se reflète dans le traité de Delamare au titre XIV intitulé «De la discipline qui doit être observée lorsque la contagion est dans une ville, bourg ou village, pour empêcher qu'elle ne communique aux autres lieux soit de la même province ou autres éloignées». En effet, l'auteur caractérise l'action de la police en ce qu' «il s'agit à présent d'examiner quelles sont les précautions que l'on doit prendre dans les lieux où ce fléau ne s'est pas encore fait sentir, pour empêcher avec le secours du Ciel qu'il n'en approche», cela avant même l'éviction des parlements, en 1720, de la lutte juridique contre les épidémies. DELAMARE, N. (1705), p. 636.

15 . V. l'arrêt du Conseil d'État du Roi du 14 septembre 1720 au sujet de la maladie contagieuse de la ville de Marseille, A.N., E 2020, fo 155, infra, p.12.

16 . Comme l'attestent les dispositions du traité de Delamare, - t. IV, Livre IV, Titre XIV - «De la discipline qui doit être observée lorsque la contagion est dans une ville, bourg ou village, pour empêcher qu'elle ne se communique aux autres lieux, soit de la même Province, ou autres plus éloignées», ce rôle en matière sanitaire est attribué en ces termes: «il est de l'ordre qu'aussitôt que ce mal paraît en quelque lieu, le principal Magistrat en donne avis à Monsieur le Procureur général du Parlement, pour y être pourvu par cette autorité supérieure à laquelle toutes les provinces qui sont dans l'étendue de son ressort doivent obéir [...]». Ce dernier cite un certain nombre d'interventions de différents parlements en matière sanitaire remontant au XVI ${ }^{\mathrm{e}}$ siècle; v. l'arrêt du 13 septembre 1533 du Parlement de Paris réglementant la peste, A. N., U415, Extraits des délibérations du Parlement de Paris concernant la peste (1522-1668), XVIII ${ }^{\mathrm{e}}$ siècle et DELAMARE, N. (1705), p. 668.

17 . A titre d'exemple, rappelons qu'avant 1720, le Parlement de Bretagne est amené à rendre des arrêts sur le fait de la contagion, les attributions relatives à la lutte contre les épidémies relevant d'une chambre de 
reconstitue son bureau de santé du 30 juillet au 30 septembre et ne rend pas moins de quarante-six arrêts pour faire face à la situation ${ }^{18}$, ce dernier enclavant totalement la province et gelant tout contact et commerce avec l'extérieur. Toutefois, n'ayant pas les finances et l'organisation nécessaires pour entreprendre une lutte provinciale contre les épidémies, le Parlement se voit confisquer cette dernière. En effet, il apparaît en 1720, lorsque le Roi décide de retirer la juridiction sanitaire - celle relative à la connaissance des crises épidémiques - aux cours souveraines, que le Parlement d'Aix n'a pas su mener une lutte efficiente contre la peste. Pire, il a provoqué un désordre dans le commerce de la province.

Le pouvoir royal s'immisce dans le partage des compétences des autorités locales par le constat qu'il dresse de leur inefficacité dans la lutte contre l'épidémie en raison des disputes entre autorités. Ces dernières sont dues aux ordres contradictoires donnés par les parlementaires aux subdélégués - souvent à l'occasion de crises épidémiques - ainsi qu'aux empiétements de fonctions et à l'entêtement des parlementaires à intervenir ${ }^{19}$, lesquels nuisent au bon fonctionnement de l'administration. L'autorité souveraine intervient par arrêts du Conseil, procédure expéditive dans son application, non soumise à enregistrement des cours à la différence des lettres patentes et exécutée sans difficultés par les autorités administratives auxquelles ils sont adressés ${ }^{20}$.

police générale, v. A.D.I.-V., fonds 1Bh et BAREAU, R. (2000), p. 196-198-440. Toujours à propos du Parlement de Bretagne, «des attributions sanitaires pouvaient relever d'une chambre de police générale; c'était le cas du parlement de Bretagne», HILDESHEIMER, F. (1996), «Les parlements et la protection sanitaire du royaume», POUMADERE, J. et THOMAS, J. (dir.), Les Parlements de Province - pouvoirs, justice et société du XV $V^{e}$ au XVIII siècle, Framespa, p. 484. Les archives des audiences de police générale sont à consulter dans la sous-série A.D.I-V., 1Bh. Aucun document, lettres de commissions de l'Intendant, ordonnance royale ou arrêt du Conseil du roi n'atteste clairement des attributions des deux organes, ce n'est que par l'éclairage de la pratique que l'on peut avancer cette hypothèse. Néanmoins, nous verrons ultérieurement qu'un arrêt du Conseil du roi évoque la question des compétences parlementaires en matière de lutte contre les épidémies, v. CADDEO, J. (2018), Pouvoirs locaux et santé publique au XVIII siècle, l'exemple de Rennes, Mémoire DEA, Histoire du droit, Rennes I, p. 124.

18. Par exemple, un arrêt du Parlement d'Aix du 31 juillet 1720 «ordonne que tout commerce soit interdit avec les habitants de la ville de Marseille, attendu le soupçon de peste, tant pour les habitants de cette ville d'Aix que pour toutes les villes et lieux de la province», v. LEBRUN, F. (1991), «Le temps de la santé publique? Derniers soubresauts de la peste», DELUMEAU, J. et LEQUIN, J. (dir.), Les malheurs des temps. Histoire des fléaux et des calamités en France, Paris, Larousse, p. 354. Sur l'action du Parlement d'Aix lors de cet événement, v. aussi PANZAC, D. (1986), «Crime ou délit? La législation sanitaire en Provence au XVIII ${ }^{\mathrm{e}}$ siècle», Revue historique, p. 39-71. En ce qui concerne les enjeux factuels et juridiques auxquels doit faire face le Parlement v. SANCHEZ, S., LECA, A., LUNEL, A. (2014), p. 138.

19 . En 1720, lors de l'épidémie de peste, le Parlement d'Aix ne communiquait pas avec les autres agents et autorités provinciales, ce qui eut pour conséquence un blocage de l'activité commerciale et politique de la Province.

20 . De ce fait, les autorités locales doivent exécuter «tout ce qui sera ordonné par lesdits Sieurs commandants et Intendants, ou par lesdits officiers municipaux (...) Mande et ordonne Sa Majesté aux Sieurs commandants et Intendants et commissaires départis dans les Provinces, et auxdits officiers municipaux, et à toutes autres qu'ils appartiendra de tenir soigneusement la main chacun en foi à l'exécution du présent arrêt», Arrêt du Conseil d’État du Roi du 14 septembre 1720 au sujet de la maladie contagieuse de la ville de Marseille, article XXVI. 
Gardons toutefois à l'esprit que cet arrêt n'est pas une réponse exclusivement motivée par les événements s'étant produits à $\mathrm{Aix}^{21}$, mais celle d'un désordre découlant des disputes entre autorités locales, couramment constaté par l'autorité royale. Cet arrêt témoigne d'une volonté délibérée d'efficacité, d'ordre et surtout d'uniformisation à l'échelle nationale de la part du pouvoir souverain ${ }^{22}$, sous couvert de centralisation. En effet, au-delà de la dépossession de la connaissance des affaires sanitaires par les parlements, le roi, par cet arrêt du Conseil d'État - ce moyen de législation soulevant lui aussi des remarques quant à la volonté royale de se faire obéir ${ }^{23}$ - s’en réserve le contentieux:

$<<$ Sa Majesté dont les vues s’étendent également aux besoins de toutes les Provinces de son royaume, désire pourvoir et prévenir en même temps les conflits de juridictions qui pourraient naître entre les amirautés, les juges de police, les officiers municipaux et autre sur une matière si importante, et qui n’admet aucun délai. Le roi étant en son conseil, de l'avis de $\mathrm{M}$. le Duc d'Orléans Régent, sans préjudice des prétentions respectives desdits officiers, et sans tirer conséquence pour leur compétence a ordonné et ordonne ce qui s'ensuit, [...] M. l'intendant se charge de prendre les mesures proportionnées [...] Sa Majesté se réserve le jugement ${ }^{24}$.>>

Les conséquences commerciales évoquées ci-dessus sont les principaux facteurs de cette mise à l'écart. Au sein de la mesure du Conseil d'État du roi, deux intérêts sont mis en balance, la liberté du commerce, de circulation d'un côté et la nécessité de protéger le royaume d'une possible contagion de l'autre. Balance entre ces deux intérêts que n’ont pas été capables d'appliquer les autorités locales de police. En effet, "plusieurs Parlements de ce Royaume ont cru devoir rendre des Arrêts, où leur zèle pour la conservation des Provinces de leur ressort les a portés à prendre des précautions surabondantes et capables non seulement d'augmenter 1 'alarme et la consternation dans le cœur des Peuples, mais encore d'interrompre le cours ordinaire du Commerce, \& de priver leur pays même, par un excès de prévoyance, des secours qui leur sont le plus nécessaires» ${ }^{25}$. Le Conseil du Roi s'en attribue alors la tâche en renfermant dans un seul arrêt «toutes les précautions qui lui sont parues nécessaires et suffisantes, pour empêcher d'un côté la communication du mal, dont elle espère que la ville de Marseille sera bientôt délivrée, pour conserver de l'autre la liberté du commerce

\footnotetext{
${ }^{21}$. Les faits de la décision l'exposent clairement : «Le Roy étant informé que le bruit de la maladie contagieuse dont la ville de Marseille est affligée, ayant rependu la crainte et l'inquiétude non seulement dans les Provinces voisines, mais dans les lieux les plus éloignés», Ibid.

${ }^{22}$. En effet, «après de premières réactions lentes et confuses, le Régent, devant la gravité du danger, fit face avec énergie : un arrêt du Conseil du 14 septembre 1720 édicte des mesures sévères pour isoler et faire vivre les régions contaminées et mettre le royaume en défense contre l'épidémie», ANTOINE, M. (2010), Le Conseil du roi sous le règne de Louis XV, Paris, Librairie Droz, p. 272.

${ }^{23}$. Cet arrêt «enlève la juridiction sanitaire aux parlements et entend unifier la législation pour tout le royaume et en réserve le contentieux au Conseil du roi. Sans doute est-ce pour éviter les discordes avec ces autorités locales jalouses de leurs prérogatives que le pouvoir royal préfère souvent recourir aux arrêts du conseil - avantage de souplesse, rapidité et simplicité car sont immédiatement applicables et emportent les mêmes effets que les lettres patentes mais incitent à un minimum de coopération entre les autorités par ces lettres patentes soumises à la vérification des lettres souveraines», SANCHEZ, S., LECA, A., LUNEL, A. (2014), p. 140; v. aussi DUMAS, A. (1956), «Michel Antoine, Le fonds du Conseil d’État du roi aux Archives nationales. Guide de recherches», Revue d'histoire de l'Église de France, t. XLII, n¹39, p. 264.

${ }^{24}$. Arrêt du 14 septembre 1720 au sujet de la maladie contagieuse dans la ville de Marseille, A.N., E 2020 , fo 1 .

${ }^{25}$. Ibid.
} 
entre les différentes Provinces de son Royaume, et veiller également à leur sûreté et à leur abondance» ${ }^{26}$. Cette sentence s'explique par le contexte économique, alors en pleine expansion au XVIII ${ }^{\mathrm{e}}$ siècle. Les cordons sanitaires établis par les parlements semblent alors trop sévères au vu des enjeux commerciaux intra regno - pour le commerce inter-provinces - et extra regno - avec les cités étrangères voisines. Néanmoins, plusieurs denrées et marchandises sont exempts de contrôle lors du transport entre provinces ${ }^{27}$ : on retrouve à ce titre les denrées rares, non périssables et différents produits alimentaires ou hygiéniques ${ }^{28}$.

Enfin, il est nécessaire d'évoquer au profit de quelle autre autorité cette substitution de compétences est opérée. L'arrêt dispose dans un premier temps qu' «il est interdit à toute autre Cours et juge, jusqu'à ce qu'autrement par elle en ait été ordonné, et ce sans avoir égard aux dispositions des arrêts rendus dans les Cours de Parlement, qui seraient donnés, \& ce sans avoir égard aux dispositions des Arrêts rendus dans les Cours de Parlements, qui seraient contraires au présent arrêt, ou qui établiraient d'autres et plus grandes précautions que celles qui sont ci-dessus marquées». C'est alors qu'elle «mande et ordonne sa majesté Commandants \& Intendants \& Commissaires départis dans les provinces et auxdits officiers municipaux et à tout autre qui appartiendra de tenir soigneusement et la main, chacun en droit à l'exécution du présent arrêt» ${ }^{29}$; c'est en effet aux intendants et commissaires de province que revient le rôle de gérer les aménagements pris lors de périodes d'épidémies. Cette substitution de compétence illustre un mouvement global de réappropriation de la police par les agents directement commis par le pouvoir central, on assiste donc à une centralisation de l'intérêt général, une affaire de tous ${ }^{30}$. En ce qui concerne le pouvoir royal, cette décision met

${ }^{26}$. À ce titre, l'article IX dispose que «Permet sadite Majesté à toutes sortes de personnes de transporter librement toutes sortes de marchandises et denrées de toutes les provinces du royaume dans celle de la Provence, et d'y conduire toutes sortes de bestiaux et ce aux lieux qui seront indiqués par lesdits commandants et intendants, par rapport aux denrées, bestiaux et marchandises qui seront nécessaires pour l'entretien et nourriture des habitants des lieux suspects», ibid., art. IX.

27 . L'article VIII donne la liste des différents produits exemptés de défense portée par l'article VII. À savoir des denrées diverses telles que et de manière exhaustive : «Les poissons secs et salés, les olives, câpres, oranges, citrons, grenades, amendes, avelines, prunes et pruneaux, figues, marrons, châtaignes, noix et autres fruits étant en barils, les huiles en cruche, et vases de terre ou tonnes, ou autres futailles, hors les peaux de boucs, les vins et autres liqueurs entonnées en futailles, les poivres, clous de girofle, gingembre, muscades, et autres épiceries, et drogues aromatiques, purgatives et préservatrices, les parfums, les cendres et soudures braillées, natrons, et savons noirs et gris (...) Le tout sans corde, emballage ni sac, et à la charge que lesdits marchandises exceptées, ne pourront être transportées de la Provence, au-delà du Verdon, de la Durance et du Rhône, qu'après qu'elles auront séjourné pendant l'espace de quarante jour sur le bord desdites rivières du côté de la mer, dans les lieux qui seront à ce destinés par lesdits commandants et intendant ou personnes par eux préposées», ibid., art. VIII.

28 . «La substitution de compétence intervient dans le cadre de l'antagonisme entre les mesures sanitaires visant à cloisonner, isoler et interdire les communications et les nécessités du décloisonnement favorable à la libre circulation du commerce. Il ne faut pas oublier en effet que l'on se situe précisément au début du décollage économique du XVIII ${ }^{\mathrm{e}}$ siècle, au moment où la croissance devient nettement perceptible et ou les entraves mises aux activités économiques par les mesures sanitaires traditionnelles deviennent proprement insupportables. C'est le fondement de l'argumentation de l'arrêt du 14 septembre 1720 qui estime que seul le pouvoir central est en mesure de juger des nécessités en ce domaine et de satisfaire à la fois aux exigences de l'économie et à celles de la santé», HILDESHEIMER, F. (1996), p. 490.

29 . Arrêt du 14 septembre 1720 au sujet de la maladie contagieuse dans la ville de Marseille, A. N., E 2020, fo 1 .

30 . Centralisation donc, de l'intérêt général face aux particularismes locaux que représentent les parlements au profit de l'intendant de la province», HILDESHEIMER, F. (1996), p. 489. La publicité de 
particulièrement en exergue la volonté souveraine de centraliser la matière sanitaire au profit des commissaires et intendants.

\section{UNE MESURE PORTANT LA DISTINCTION ENTRE PREVENTION ET LUTTE CONTRE LA MALADIE CONTAGIEUSE}

Si l'on s'en tient strictement à la lettre de l'arrêt, le Conseil du roi met alors en place, de manière purement factuelle, un cordon sanitaire et organise une procédure de quarantaine. L'enjeu sous-jacent de ces deux mesures réside dans le fait qu'elles traduisent une volonté de préservation de la santé plutôt que la manifestation d'une volonté d'éradication de la maladie. En effet, la mise en place de cordons sanitaires et règlements concernant le phénomène épidémique n’ont alors pas pour but son éradication mais de limiter sa propagation, la lutte étant entendue comme une action des autorités publiques aux fins d'éradication. Il faut alors reprendre en détail les définitions de ces instruments sanitaires pour en saisir les conséquences sur l'action des parlements provinciaux.

Dans un premier temps, c'est donc un cordon sanitaire qui est mis en place; ce dernier consiste d'une part à isoler la ville de Marseille, contaminée par la peste, et à en limiter les échanges avec les autres cités. L'article I de l'arrêt prescrit alors que «Sa majesté fait très expresse inhibitions et défenses aux habitants de la ville de Marseille et à tous ceux qui demeureront dans l'étendue des limites ou barrières qui ont été marquées aux environs de laditte ville par les commandants et intendants de Provence, de sortir hors desdites limites et barrières, et d'en transporter aucunes marchandises ni denrées, à peine de la vie» ${ }^{31}$. S'ensuit une délimitation du cordon sanitaire via une interdiction de circulation des personnes et marchandises. De ce fait, «Défend pareillement Sa Majesté, sous telles peines qu'il appartiendra, aux habitants de Provence, demeurant hors desdites limites, et à tous ceux des Provinces voisines d'aller dans les lieux compris dans lesdites limites, si ce n'est en vertu d'un ordre exprès et par écrit des commandants ou intendants susdits; et à l'égard de ceux qui en seraient allé avant la publication du présent arrêt, ou qui pourraient y aller au préjudice des défenses, ils ne pourront en sortir qu'à la charge de faire quarantaine dans les lieux qui seront désignés à cet effet par lesdits commandants ou intendants, ou personnes par eux préposées» ${ }^{32}$. De plus, l'arrêt fait «très expresses inhibitions et défenses à toutes sortes de personnes de quelque état et condition qu'elles soient, de transporter aucunes marchandises ou denrées de quelque nature et qualité qu'elles puissent être au-delà desdites rivières du Verdon, de la Durance et du Rhône et aux commis établis par l'adjudicataire de ses fermes aux environs desdites rivières, de les laisser passer le tout à peine de la vie» ${ }^{33}$. Dans ces quelques lignes, c’est bien la dimension de préservation de la santé qui est mise en exergue. La finalité du cordon sanitaire n’étant pas d'éradiquer l'épidémie de peste mais bien de prévenir, de contenir la diffusion du mal.

l'arrêt nous informe sur la portée de la mesure : l'article IV de la décision prévoit qu' «afin que personne ne puisse prétendre cause d'ignorance des défenses portées par les articles précédents, il sera publié à toutes les entrées et chemins où il n'y aura point de barrière, des poteaux avec des inscriptions qui contiendront les défenses ci-dessus marquées», Arrêt du 14 septembre 1720 au sujet de la maladie contagieuse dans la ville de Marseille, art. IV.

31 . Arrêt du 14 septembre 1720 au sujet de la maladie contagieuse dans la ville de Marseille, $\mathrm{f}^{\circ} 1$, art. I.

32 . Ibid.

33 . Ibid. 
L'exemple de la quarantaine est lui aussi éloquent; en effet, cette dernière est une technique de prévention sanitaire utilisée très tôt dans les sociétés et se manifeste par la création de lazarets aux abords des côtes contaminées. À Marseille, le premier lazaret aurait été fondé en 1526 puis, après des transferts successifs, il fut établi en 1663 immédiatement à l'ouest de la ville ${ }^{34}$.

En l'espèce l'article III prévoit qu' «il ne sera permis à aucun habitants dudit pays de Provence, ni de ceux des autres pays qui y sont actuellement, encore qu'ils soient hors des limites dont il a été fait mention dans l'article premier, de passer les rivières du Verdon, de la Durance ou du Rhône sans avoir fait la quarantaine pendant le temps qui leur sera prescrits par les lesdits commandants et intendants et ce dans les lieux qui seront désignés par lesdits commandants et intendants, ou par ceux qu'ils auront préposés à cet effet [...]» ${ }^{35}$. Une fois le périmètre délimité, l'arrêt détaille les conditions et délais de la quarantaine; ainsi, «si le vaisseaux n'est pas muni de lettres de santé, ou si les lieux dans lesquels les lettres lui auront été accordées sont simplement suspects, sans aucuns avis certain qu'il y ait de la contagion, lesdits officiers municipaux seront tenus d'observer et faire observer les formalités prescrites par les articles XVII et autres suivants; permet néanmoins Sa Majesté aux Sieurs Intendants et Commissaires départis dans les Provinces d'abréger ou proroger la quarantaine ainsi qu'ils le jugeront à propos» ${ }^{36}$. Deux solutions sont alors envisagées: "Si après l'expiration de la quarantaine il n'a paru aucun indice de mal contagieux, lesdits officiers permettront l'entrée et la libre communication et disposition du vaisseaux, de l'équipage et des marchandises (...) Si pendant le cours de la quarantaine ceux qui y auront été assujettis sont attaqués du mal contagieux, lesdits officiers municipaux leur feront faire une seconde quarantaine, qui commencera du jour que le mal se sera déclaré» ${ }^{37}$.

Au vu de ces extraits, il est certain que l'autorité n'essaie pas ici d’éradiquer l'épidémie, mais s’occupe de potentiels porteurs de cette dernière. C’est justement parce qu'elle prend en compte ce danger «potentiel» que l'on peut affirmer que la procédure de quarantaine et la mise en place d'un cordon sanitaire apparaissent comme des moyens de prévention de l'épidémie.

\section{UNE MESURE NE REMETTANT PAS EN CAUSE LA COMPETENCE SANITAIRE DES PARLEMENTS DE PROVINCE}

C'est parce qu'elle distingue prévention et lutte que cette mesure nous éclaire sur les compétences de police des parlements. Concernant la réception par les différentes cours provinciales de cette dispositions les rébellions du Parlement d'Aix ${ }^{38}$

\footnotetext{
34 . Les lazarets sont des établissements où sont isolées les personnes ou les marchandises contaminées ou susceptibles d’avoir été contaminées par une maladie épidémique, v. LAGET, P.-L. (2002), «Les lazarets et l'émergence de nouvelles maladies pestilentielles au XIX et au début du XX ${ }^{\mathrm{e}}$ siècle», In Situ, n²2, p. 4. Sur l'histoire des lazarets, v. aussi PANZAC, D. (1990), Quarantaines et lazarets. L'Europe et la peste d'Orient, du XVII ${ }^{e}$ au XX $X^{e}$ siècles, Edisud, Aix-en- Provence, p. 200 et PRETO, P. (1978), Peste e società a Venezia nel 1576, Vicenza, Neri Pozza, p. 80.

${ }^{35}$. Arrêt du 14 septembre 1720 au sujet de la maladie contagieuse dans la ville de Marseille, f ${ }^{\circ} 1$, art III.

36 . Ibid., art. XXV.

37 . Ibid., art. XXIV et XXIII.

38 . Le Parlement d'Aix ne se laisse pas faire et «la réaction des parlementaires aixois devant ce texte qui les dépouillait de leur attribution traditionnelle fut, bien sûr, d'en refuser l'enregistrement et, en outre,
} 
fournissent, contre son gré, des précisions sur l'étendue concrète de l'interdiction: dans un arrêt du 3 octobre 1722, le Conseil du Roi explique que «Sa majesté ayant interdit audit Parlement la connaissance de tout ce qui concernait la contagion qu'il avait attribué aux commandants de la province, cette cour se trouvait sans pouvoir ni juridiction pour un fait qu'il avait été décidé par lesdits Commandants [...] fait sa Majesté défenses audit Parlement de prendre à l'avenir aucune connaissance de l'exécution des jugements rendus concernant la contagion par les commandants de la province ou en conséquence de leurs ordres, circonstances et dépendances à peine de nullité, cassation de procédures et de tous dépens dommages et intérêts» ${ }^{39}$. De son côté, en 1784, le Parlement de Paris invoque des «traditions juridiques», terme bien modéré, pour justifier son inaction dans ce domaine au profit de l'intendant43. Quant au Parlement de Bretagne, il n'y a pas à proprement parler de mouvement de rébellion à partir de 1720-1722, qui aurait pu se révéler par des remontrances à ce propos. Et justement, on peut interpréter cette absence de sursaut juridique de telle manière qu'il existerait, à la disposition des Cours souveraines, un autre procédé pour contourner la décision du Conseil.

Il semble alors essentiel de nuancer en attribuant une portée plus spécifique à l'arrêt étudié ci-dessus. En effet, on pose à juste titre que le pouvoir souverain a enlevé la matière sanitaire aux parlements du royaume, par conséquent les parlements ne devraient plus, à partir de 1720, rendre d'arrêts de police concernant directement la matière sanitaire. Or, on s'aperçoit, que si l'arrêt est interprété de la sorte, les parlements contournent cette interdiction en agissant en amont de la maladie et en prenant des mesures préventives ${ }^{40}$, par exemple via la police des rues. En effet, cette dernière ne concerne pas spécifiquement la lutte ${ }^{41}$ - supposant la présence de la maladie

d'en faire détruire les exemplaires imprimés et diffusés dans le ressort de la Cour», HILDESHEIMER, F. (1996), p. 487.

39 . Arrêt du 3 octobre 1722, A.N., E2020, fo 296.

40 . À Paris en 1784, le Parlement de Paris, lors d'une épidémie à Argenteuil, cède sa place à l'Intendant de Paris Bertier de Sauvigny invoquant les «traditions juridiques» qu'il ne souhaite pas bouleverser malgré sa compétence reconnue et réservée. L’intendant prend donc le relais : «des raisons juridiques déterminent le Parlement à ne pas intervenir malgré l'invitation de l'Intendant. [...] Ici donc le Parlement dont la compétence était à la fois reconnue et réservée l'a-t-il abandonnée à l'Intendant à cause du respect des usages qu'il ne prend pas sur lui de bouleverser. En l'espèce, l'Intendant joint donc à sa compétence naturelle en matière de travaux publics la police dont le Parlement ne veut pas se charger parce qu'elle est tenue en échec par la tradition juridique. Plutôt que de s'estimer compétent mais de refuser d'agir en raison d'un scrupule, sans doute honorable, mais qui aurait pour effet de paralyser la lutte contre l'épidémie, le Parlement abandonne le terrain». Ici, P. Payen explique l'inaction du Parlement de Paris par des «traditions juridiques», nous ne savons pas si ce terme vient de l'auteur ou de la Cour souveraine mais il ne semble pas adapté dans le sens où les raisons de non interventionnisme sont beaucoup plus fortes, ce sont des mesures juridiques prises par le Conseil d'Etat du Roi - toujours en rapport avec l'arrêt du 14 septembre 1720 - qui justifient que le Parlement laisse l'Intendant s'occuper des questions de lutte épidémiques, v. PAYEN, P. (1997), pp. 143- 144.

41. Cet exemple est valable pour les autres Parlements du royaume de France, par exemple en 1721 le maréchal de Villars, gouverneur de Provence, prend part à un Conseil chez le Prince - le terme exact étant un «conseil de peu de personnes» - où il est menée une réflexion sur les désordres de la peste et des moyens de l'empêcher de s'étendre», sans aucune répression de la part du pouvoir central ce qui atteste bien que les mesures de prévention sont toujours du ressort des Parlements, v. ANTOINE, M. (2010), p. 27. En Bretagne, c'est aussi le cas lorsque le Parlement reporte la rentrée des classes pour cause de dysenterie, justifiant ces mesures du fait que l' «on ne peut prendre trop de précaution pour l'en garantir», ici la dimension préventive est encore soulignée, la Cour souveraine n’a pas vocation à éradiquer la maladie mais bien à aider les habitants à s'en prévenir en amont, v. la remontrance du procureur général du roi, Arrêt de la Cour du 12 octobre 1756, A.D.I.-V., 1Ba69 cité par BAREAU, R. (2000), p. 340. 
- mais plutôt la prévention lorsqu'elle agit sur les facteurs tant de déclenchement que de diffusion. S'offrent alors à nous deux possibilités: soit l'arrêt du 14 septembre 1720 interdit aux parlements, d'un point de vue matériel, uniquement l'édiction de mesures prises dans le cadre de la lutte contre l'épidémie et non l'édiction de mesures de prévention de cette dernière, les Cours souveraines agiraient donc dans leur bon droit lorsqu'elles agissent sur le volet préventif; soit cet arrêt concerne l'édiction de mesures sur tout ce qui a trait à la contagion - sa prévention comprise - et là s'opérerait un truchement de la part des parlements afin de continuer à réglementer les aspects liés à l'épidémie ${ }^{42}$. La première solution semble la plus à même d'expliquer l'intervention des parlementaires en matière sanitaire, d'autant plus que, comme vu précédemment, la police lato sensu ainsi que la police sanitaire sont les piliers de la prévention sanitaire, ainsi la décision souveraine d'enlever les compétences aux autorités locales en ayant l'exercice serait déraisonnable, tant sur le plan théorique que pratique, en termes d'efficacité.

Malgré le champ d'application très large de la police, le rôle des pouvoirs locaux s’inscrit dans une optique que l'on qualifie de "préventive» ${ }^{43}$, effectuée en vue d'une protection sanitaire de la province consistant non en une éradication, mais en une réduction de l'exposition des habitants aux dangers que sont les maladies présentes ${ }^{44}$. On peut donc prétendre que le parlement a contourné la mesure royale pour réactiver sa compétence grâce à la prévention. Cette prévention sanitaire est alors affaire des pouvoirs locaux, d'une part au vu de leur rôle étudié précédemment en matière de prévention et, d'autre part, au vu de leur la place au sein de la lutte contre les épidémies.

A la lecture de ces quelques lignes, n’a-t-on pas là une preuve supplémentaire que le pouvoir royal poursuit la même politique ancienne consistant à conserver les institutions en les plaçant en concurrence les unes avec les autres, lui permettant alors de s'assurer de l'applicabilité des mesures et de rester l'arbitre des querelles institutionnelles? Dans le cadre de cette monarchie que l'on qualifie volontiers d'«administrative», mise en place dès le règne de Louis XIV, l'autorité royale ne se contente plus de ses fonctions de justice et de ses attributions militaires mais étend son action dans des secteurs plus ciblés dont la santé des sujets fait partie. Pour ce faire, le roi n'a pas d'autre choix que de déléguer son pouvoir d'exécution sans pour autant dénaturer l'influence de l'Etat monarchique, le souverain gardant le pouvoir de décision.

\footnotetext{
42 . Désormais entendue comme «l'éradication de l’épidémie» et dévolue à l’intendant.

43 . Cette question se posant pour la première fois ici, le parti pris est celui d'opter pour la première solution qui interpréterait l'arrêt du 14 septembre 1720 comme distinguant la prévention de la lutte effective. Cette distinction entre lutte préventive et lutte effective en tant qu'éradication se reflète dans le traité de Delamare au titre XIV intitulé «De la discipline qui doit être observée lorsque la contagion est dans une ville, bourg ou village, pour empêcher qu'elle ne communique aux autres lieux soit de la même province ou autres éloignée». En effet, l'auteur caractérise l'action de la police en ce qu' «il s'agit à présent d'examiner quelles sont les précautions que l'on doit prendre dans les lieux où ce fléau ne s'est pas encore fait sentir, pour empêcher, avec le secours du Ciel, qu'il n'en approche», DELAMARE, N. (1705), (N.), p. 636.

44 . En guise d'exemple de cette spécialisation des pouvoirs locaux en tant qu'autorités publiques chargées de la police préventive on constate, au-delà du cadre breton, que «la Lieutenance générale de police de Paris, au siècle des Lumières, développa une "politique policière de santé" urbaine en direction des habitants. Dépassant la lutte contre les épidémies, elle devint préventive et s’intéressa aux liens entre les êtres humains et leur environnement. Elle mobilisa pour cela des savoirs urbains de pointe, en produisit de nouveaux, et intégra dans sa sphère la Société Royale de Médecine, qui lui permit d'agir directement sur la matérialité urbaine pour améliorer la santé des Parisiens», VIDONI, N. (2015), p. 97.
} 
C'est dans cet esprit de délégation contrôlée du pouvoir que la santé publique va être administrée au XVIII ${ }^{\mathrm{e}}$ siècle. Quoi qu'essentielle dans la formation de cette administration, l'action des pouvoirs locaux précède toujours la volonté du souverain. Les institutions locales agissent ainsi tantôt sous l'égide royale, tantôt avec une relative autonomie, elle-même concédée.

\section{BIBLIOGRAPHIE}

Ouvrages

ANTOINE, M. (2010), Le Conseil du roi sous le règne de Louis XV, Paris, Librairie Droz.

BAREAU, R. (2000), Les arrêts de règlement du Parlement de Bretagne, Thèse, Droit, Rennes I, t. II.

CADDEO, J. (2018), Pouvoirs locaux et santé publique au XVIII siècle, l'exemple de Rennes, Mémoire DEA, Histoire du droit, Rennes I.

CAPLET, E.-A. (1898), La Peste à Lille au XVII ${ }^{e}$ siècle, Lille, Le Bigot frères.

DELAMARE, N. (1705), Traité de la police, où l'on trouvera l'histoire de son établissement, les fonctions et les prérogatives de ses magistrats; de toutes les loix et règlements qui la concernent, Paris, J. et P. Cot, t. I.

DELHUMEAU, J. et LEQUIN, Y. (1993), Les malheurs des temps. Histoire des fléaux et des calamités, Paris, Larousse.

FERRIERE, C. de (1717), Dictionnaire de droit et de pratique, Paris, Babuty fils.

GAFFIOT, F. (1934), Dictionnaire latin-français, Paris, Hachette.

MESTRE, J.-L. (1985), Introduction historique au droit administratif, Paris, PUF.

PANZAC, D. (1990), Quarantaines et lazarets. L'Europe et la peste d'Orient, du XVII au $X X^{e}$ siècles, Aix-en- Provence, Edisud.

PAYEN, P. (1997), Les arrêts de règlement du Parlement de Paris au XVIII ${ }^{e}$ siècle. Dimension et doctrine, Paris, PUF.

PRETO, P. (1978), Peste e società a Venezia nel 1576, Vicenza, Neri Pozza.

SANCHEZ, S., LECA, A., LUNEL, A. (2014), Histoire du droit de la santé, Bordeaux, Les études hospitalières, Collection Intempora.

SAINT- BONNET, F., SASSIER, Y. (2015), Histoire des institutions avant 1789, Paris, Domat Droit Public.

SOURNIA, J.-C. (1984), Les épidémies dans l'histoire de l'Homme, Paris, Flammarion.

$\underline{\text { Articles }}$

BOULET-SAUTEL, M. (1980), «Police et administration en France à la fin de l'Ancien Régime. Observations terminologiques: Histoire comparée de l'administration (IV ${ }^{\mathrm{e}}-\mathrm{XVIII} \mathrm{e}^{\mathrm{e}}$ siècle)», Actes du XV colloque historique franco-allemand, Tours 1977, Munich, pp. 47-51.

BRUNET F. (2009), «Introduction», ROUSSELET-PIMONT, A., BONIN, P., GARNIER, F. et HUMBERT, M. (dir.), Études d'histoire du droit rassemblées en l'honneur d'Albert Rigaudière, Paris, Economica, pp. 16-24.

DUMAS, A. (1956), «Michel Antoine, Le fonds du Conseil d'État du roi aux Archives nationales. Guide de recherches», Revue d'histoire de l'Église de France, t. XLII, n¹39, pp. 264-267.

FAURE, O. (1997), "Les stratégies sanitaires», in GRMEK, M. (dir.), Histoire de la pensée médicale en Occident, Paris, t. II, pp. 279-298. 
HILDESHEIMER, F. (1996), «Les parlements et la protection sanitaire du royaume», POUMADERE, J. et THOMAS, J. (dir.), Les Parlements de Province pouvoirs, justice et société du XV au XVIII ${ }^{e}$ siècle, Framespa, 1996, pp. 443467.

LAGET, P.-L. (2002), «Les lazarets et l'émergence de nouvelles maladies pestilentielles au XIX ${ }^{\mathrm{e}}$ et au début du $\mathrm{XX}^{\mathrm{e}}$ siècle», In Situ [En ligne], n²2, 2002, mis en ligne le 23 avril 2012.

LEBRUN, F. (1991), «Le temps de la santé publique? Derniers soubresauts de la peste», DELUMEAU, J. et LEQUIN, J. (dir.), Les malheurs des temps. Histoire des fléaux et des calamités en France, Paris, Larousse, pp. 203-277.

LECA, A. (2003), «La notion d'épidémie dans l'histoire des idées», Avant-propos au colloque d'Aix-en-Provence des 28-29 novembre 2002, «Le risque épidémique», Actes II Colloque national Droit, Histoire, Médecine et Pharmacie, Collection Droit de la santé, Aix-en-Provence, Presses Universitaires Aix-Marseille, pp.1324.

PANZAC, D. (1986), «Crime ou délit? La législation sanitaire en Provence au XVIII ${ }^{\mathrm{e}}$ siècle», Revue historique, pp. 39-71.

REVEL, J. (1970), «Autour d’une épidémie ancienne: la peste de 1660-1670», Revue d'histoire moderne et contemporaine, t. XVII, n²4, octobre-décembre, pp. 953983.

VIDONI, N. (2015), «Protéger la santé des Parisiens au XVIII ${ }^{\mathrm{e}}$ siècle: savoirs urbains et action policière», Histoire, médecine et santé, nº, pp. 97-110. 


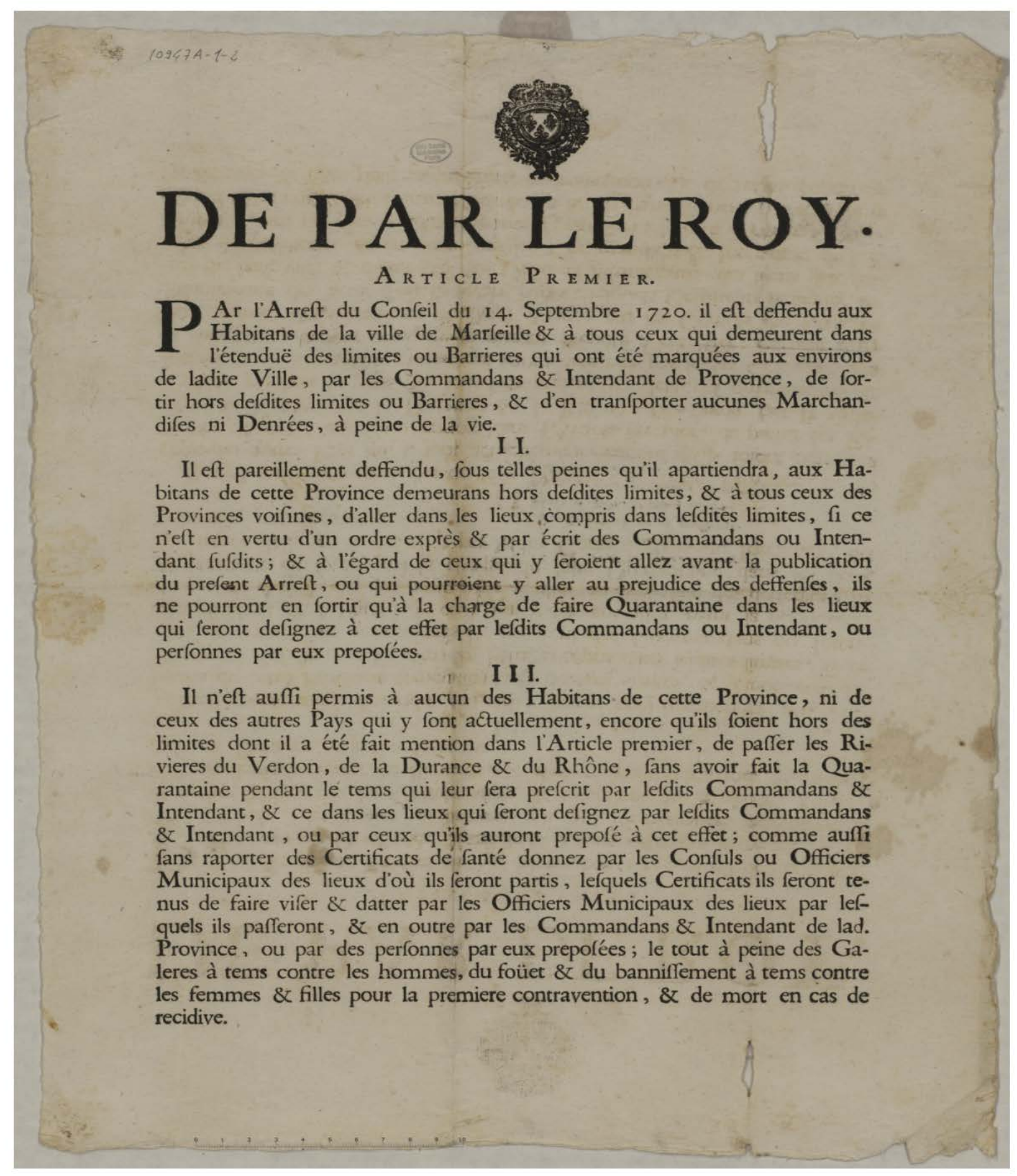

Revista de Estudios Jurídicos n²0/2020 (Segunda Época)

ISSN-e 2340-5066. Universidad de Jaén (España)

Versión electrónica: rej.ujaen.es 